
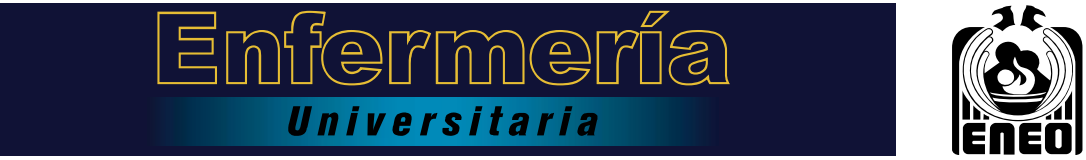

\title{
Actitud de los estudiantes de enfermería hacía los adultos mayores evaluada con las escalas de Osgood y Kogan.
}

\section{Attitudes of nursing students towards old patients as measured by Osgood and Kogan scales}

\section{Atitude dos estudantes de enfermagem face aos idosos utilizando as escalas de Osgood e Kogan}

\author{
I.A. Hernández-Vicente*, O. Moguel-Ortíz, M. Hernández- \\ Ramírez, I. Lumbreras-Delgado, J.A. Banderas-Tarabay. \\ Coordinación en Enfermería, Facultad de Ciencias de la Salud, Universidad Autónoma de Tlaxcala, Tlaxcala, \\ México \\ Recibido: 1 agosto 2017 \\ Aceptado: 13 marzo 2018
}

\section{Resumen}

Introducción: El incremento de la población de adultos mayores, requiere de particular atención y planeación en el área de la salud, así como considerar las actitudes hacia ellos, principalmente por parte de los estudiantes de enfermería quienes los cuidarán después de graduarse.

Objetivo: Identificar la actitud que tienen los estudiantes de enfermería hacia el adulto mayor y el interés que muestran en cuidar a esta población.

Material y métodos: Estudio descriptivo, cuantitativo y trasversal realizado por medio de una encuesta a una muestra representativa de 283 estudiantes de ambos sexos de la carrera de enfermería. El instrumento de investigación se aplicó para determinar la actitud que tienen los estudiantes hacia el adulto mayor con la utilización de dos escalas: el diferencial semántico de Osgood y la de Kogan.

Resultados: Con base en la escala de Osgood el 37.7\% de los estudiantes tienen una actitud negativa hacia el adulto mayor y con la escala de Kogan se obtuvo el 47\% para esta categoría. En promedio, la actitud de los estudiantes fue valorada como regular, y es el sexo femenino quien tiene más actitudes positivas, independientemente de la escala utilizada. Al 51.9\% le gustaría desempeñarse en el área gerontológica. Conclusiones: Los estudiantes de enfermería tienen más actitudes negativas relacionadas principalmente a la fragilidad y necesidades de cuidado que requiere el adulto mayor. Es necesario revisar los programas educativos en enfermería para que el aprendizaje y la comunicación orienten al estudiante desde el inicio de la carrera, para incrementar las experiencias y actitudes positivas hacia este grupo social.

*Autor para correspondencia, correo electrónico: irmalehv@hotmail.com (I.A. Hernández-Vicente) 
Palabras clave: Actitud; escala de Kogan; escala de Osgood; estudiantes de enfermería; adulto mayor; México.

\begin{abstract}
Introduction: The increasing older adult population requires special health attention and planning which should include considerations on the attitudes which nursing students demonstrate while taking care of these individuals.

Objective: To identify the attitudes and caring interest which nursing students have towards older adults. Material and methods: This is a descriptive, quantitative, and transversal study which used a survey on a representative sample of 283 nursing students of both sexes to determine their attitudes towards older adults in terms of two scales: the Osgood Semantic differential and the Kogan scale.

Results: In terms of the Osgood's scale, $37.7 \%$ of students showed a negative attitude towards older adults, while in terms of Kogan's scale, $47 \%$ demonstrated a negative attitude. In average, the students' attitude was assessed as regular, though the female students exhibited a more positive attitude regardless of the scale. $51.9 \%$ of the sample stated they would accept working in the area of gerontology. Conclusions: Nursing students in general show more negative than positive attitudes towards caring older adults, particularly regarding issues of fragility and special needs. Strengthening the nursing education programs in terms of improving the experiences and attitudes of nursing students towards the older adult population is recommended.
\end{abstract}

Keywords: Attitude; Kogan scale; Osgood scale; nursing students; aged; Mexico.

\title{
Resumo
}

Introdução: O incremento da população de idosos requer de particular atenção e planejamento na área de saúde, assim como considerar as atitudes face a eles, principalmente por parte dos estudantes de enfermagem quem os cuidaram depois de formar-se.

Objetivo: Identificar a atitude que têm os estudantes de enfermagem face ao idoso e o interesse que mostram para cuidar a esta população.

Material e métodos: Estudo descritivo, quantitativo e transversal realizado por meio de uma enquete a uma amostra representativa de 283 estudantes de ambos os sexos da carreira de enfermagem. O instrumento de pesquisa aplicou-se para determinar a atitude que têm os estudantes face ao idoso com a utilização de duas escalas: o diferencial semântico de Osgood e a de Kogan.

Resultados: Com base na escala de Osgood o $37.7 \%$ dos estudantes têm uma atitude negativa face ao idoso e com a escala de Kogan obteve-se o $47 \%$ para esta categoria. Em média, a atitude dos estudantes foi valorizada como regular, e foi o sexo feminino quem teve mais atitudes positivas, independente da escala utilizada. O 51.9\% gostaria de desenvolver-se na área gerontológica.

Conclusões: Os estudantes de enfermagem têm mais atitudes negativas relacionadas, principalmente à fragilidade e necessidades de cuidado que requer o idoso. É necessário revisar os programas educativos em enfermagem para que a aprendizagem e a comunicação orientem o estudante desde o início da carreira para incrementar as experiências e atitudes positivas face a este grupo social.

Palavras chave: Atitude; escala de Kogan; escala de Osgood; estudantes de enfermagem; idoso; México.

\section{Introducción}

Los cambios demográficos en la población adulta requieren de profesionales preparados en enfermería para su cuidado. Se estima que para el 2050 la proporción de adultos mayores (AM) se duplicará del $11 \%$ al 22\%, lo que representa dos billones de personas mayores de 60 años $^{1}$. La Encuesta Nacional de Salud y Nutrición ${ }^{2}$ reportó que más de 500 mil AM viven en situación de dependencia y requieren 
de cuidados. En México, actualmente se estima que existe un adulto mayor por cada 13 personas y dentro de medio siglo la relación será de 1:4; para el 2030, el 18.7\% de las mujeres corresponderá a personas de 60 o más años, y 16.2\% de los hombres en la misma condición. En los próximos 30 años en el Estado de Tlaxcala, la tasa de dependencia de los adultos mayores pasará de 0.9 a 1.4 por cada 100 personas en edad productiva, y existirán 37 adultos mayores por cada 100 jóvenes ${ }^{3}$. Se calcula que, en los próximos 20 años, el $75 \%$ de los cuidados brindados por enfermería estarán dirigidos a las personas mayores de 65 años.

El aumento de esta población genera mayor demanda de los servicios especializados y un alto impacto en el costo de la atención a la salud ${ }^{4}$, lo que ocasiona agentes estresores en la sociedad ${ }^{5}$. La OMS, reconoce que en América Latina y el Caribe, las deficiencias del sistema de atención para los AM incluye la mala capacitación del personal a su cuidado, la sobrecarga de trabajo, la mala atención y el deterioro de las instalaciones dónde se encuentran ${ }^{6}$. Esto hace difícil la interacción entre el AM y el cuidador, a menudo se desconocen los valores propios de esta población, lo que se constituye en maltrato, y/o discriminación, esto expresa que no se valora a dicho sector de la población.

La mayoría de los estudiantes de enfermería trabajarán en un futuro con adultos mayores, es urgente y necesario prepararlos adecuadamente para mejorar sus conocimientos, actitudes y habilidades con el propósito de proveer cuidados de calidad. Estudios realizados en países como Holanda ${ }^{7}$ y en los Emiratos Árabes Unidos ${ }^{8}$ sobre conocimiento y actitudes, muestran que la mayoría de los estudiantes de enfermería tienen actitudes negativas hacia el AM, en contraste con estudios en España ${ }^{9}$, Taiwan ${ }^{10}$ y Suiza ${ }^{11}$, donde las actitudes son más positivas. Dichas actitudes pueden deberse a las experiencias observadas en otros profesionales y por el tipo de educación que tuvieron durante su preparación universitaria $^{7}$. La teoría de la acción razonada, postulada por Fishbein y Azjen ${ }^{12}$, sugiere que las actitudes pueden influenciar el comportamiento de un individuo, ya que las personas con una actitud positiva tendrán pensamientos más positivos hacia los demás. Medir las actitudes de los estudiantes hacia el adulto mayor es esencial para identificar y preparar mejores profesionales para el cuidado y atención del adulto mayor ${ }^{13}$.

Las actitudes negativas, voluntarias e involuntarias, como el rechazo, maltrato, gestos, poca comunicación, restricción terapéutica, entre otros, afectan la salud y calidad de vida del AM, generan depresión, baja autoestima, incomprensión e inseguridad en la terapéutica indicada; esto induce al AM a cambiar su conducta sobre los tratamientos asignados, alterar la dosis de los medicamentos, horarios y forma de ingerirlos, así como no seguir las indicaciones médicas para un eficaz tratamiento ${ }^{14}$.

Las actitudes constituyen valiosos elementos para predecir los diferentes tipos de conductas. La actitud se refiere a un sentimiento a favor o en contra de un objeto social, el cual puede ser una persona, un hecho social, o cualquier producto de la actividad humana. Ya sean positivas o negativas, influyen en las personas, son las actitudes negativas las que mayores consecuencias traen a los $\mathrm{AM}^{15}$; estas van desde las muy sutiles, como una frase incisiva y humillante, hasta el abuso físico, emocional, psicológico, financiero, sexual, así como desatención, la negación a brindarle ayuda, abandono, intimidación, injurias orales, golpes y episodios criminales; aspectos que son causantes de nerviosismo, temores, aislamiento e inactividad, entre otros ${ }^{5}$. El aprendizaje sobre el cuidado se desarrolla desde muy temprano cuando se interactúa con las personas a las que se cuida, y se requiere de preparación, sensibilidad y pensamiento crítico $^{16}$.

Para determinar la actitud que tiene un sujeto hacia el adulto mayor se utiliza la escala de actitudes hacia el adulto mayor de Kogan ${ }^{17}$, y para evaluar la percepción que tiene un sujeto hacia el adulto mayor se utiliza la escala de diferencial semántico de Osgood $^{18}$.

Muchos trabajadores de la salud piensan que los pacientes mayores son demasiado viejos para aprender o comprender, para tomar decisiones sobre su cuidado y para ser cuidados ${ }^{15}$. En enfermería como otras ciencias, la construcción de un mundo donde los AM, de hoy y de mañana, posean atención digna y decorosa, es importante para derribar las barreras y estereotipos sociales, que se pueda 
avanzar hacia una cultura de tolerancia, respeto y aceptación ${ }^{19}$. A la fecha se conoce muy poco en México sobre el conocimiento y las actitudes que tienen los estudiantes de enfermería hacia el adulto mayor al inicio de su carrera. En este contexto se reflexiona sobre las actitudes que podrían manifestar durante la atención que brindarán al AM en su vida profesional. Es así como el propósito de este trabajo es identificar la actitud de los estudiantes de la licenciatura en enfermería hacia los AM a través de la escala de Kogan y la de Osgood, además, determinar su interés por el cuidado del AM al terminar su carrera.

\section{Materiales y Métodos}

Población de Estudio. La presente investigación es un estudio descriptivo y trasversal, realizado en la Escuela de Enfermería de la Facultad de Ciencias de la Salud de la Universidad Autónoma de Tlaxcala, México, durante el mes de mayo del 2015. El universo de estudio se constituyó por 793 estudiantes de la licenciatura que fueron invitados a participar voluntariamente y a los cuales se les informó previamente del propósito de la investigación. Se calculó una muestra probabilística, estratificada al azar de 283 sujetos de ambos sexos, a través de la fórmula para poblaciones finitas con un IC del 95\% y un error muestral estimado del $5 \%$.

Diseño y aplicación del cuestionario. Se realizó una encuesta a los estudiantes de enfermería de primero a octavo semestre, del turno matutino y vespertino, se excluyó a quienes no desearon participar en el estudio. El tiempo promedio de respuesta fue de 10 minutos.

Se registraron las características sociodemográficas de cada estudiante, como son: la edad, sexo, grado escolar, con quien vive, si en su familia hay personas mayores de 60 años; en forma adicional a las escalas, se exploró si ha vivido con personas mayores de 60 años, si durante su carrera ha tratado temas sobre el adulto mayor, si le interesaría conocer más acerca de este y, finalmente si le gustaría desempeñarse en el área gerontológica.

Se determinó la actitud del estudiante de enfermería a través de la escala de actitud hacia el adulto de Kogan ${ }^{17}$, que consta de 34 enunciados, 17 de ellos tienen enunciados positivos y 17 negativos; el instrumento ha sido traducido y validado a varios idiomas ${ }^{7,10,20}$ incluido el español ${ }^{15}$ y el cual fue utilizado en este estudio con una confiabilidad del coeficiente alfa de Cronbach de 0.82. Se utilizó una escala de valores tipo Likert dividida en seis categorías: 1. Muy en desacuerdo, 2. Un poco en desacuerdo, 3. En desacuerdo, 4. De acuerdo, 5. Un poco de acuerdo y 6. Muy de acuerdo. La calificación mínima (negativa) y máxima (positiva) fueron de 34 y 204, respectivamente y la media neutral de 136.

Para evaluar la percepción de los estudiantes de enfermería hacia el adulto mayor se utilizó la escala de diferencial semántico de Osgood $^{18}$, que mide el significado connotativo de estímulos visuales y verbales; los estímulos o experiencias (adjetivos) son bipolares (16) y van de la opinión positiva a la negativa (p.ej. de innovador a rutinario) y otras preguntas van de la opinión negativa a la positiva (p.ej. de improvisado a planificado). La puntuación máxima posible (7) es considerada favorable al 100\%, y la puntuación mínima (1 punto) como menos favorable con un $0 \%$. El instrumento utilizado ha sido validado en varios estudios ${ }^{18}$, con una confiabilidad de 0.83 con la escala tipo Thurstone. A partir de esos dos extremos se calculó la puntuación en valores porcentuales para cada pregunta. La encuesta fue aplicada por pasantes de enfermería en su servicio social con apoyo de docentes previamente capacitados sobre el manejo y registro del instrumento.

Consideraciones bioéticas. La presente investigación es no invasiva por lo que no existe riesgo para los sujetos de estudio. La información obtenida bajo consentimiento informado, se mantuvo totalmente confidencial. El estudio fue realizado y autorizado por el comité de ética de la Facultad de Ciencias de la Salud y el de Enfermería, de la Universidad Autónoma de Tlaxcala, México, se siguieron los lineamientos propuestos en la declaración de Helsinki ${ }^{21}$ y de acuerdo con el reglamento de la ley general en materia de investigación en salud ${ }^{22}$.

Análisis Estadístico. Se realizó el análisis descriptivo de las variables con medidas de tendencia central, desviación estándar (DE) y percentiles. Las variables cualitativas y cuantitativas se analizaron por medio 
de frecuencias y porcentajes. Para determinar si existía relación de dependencia entre el sexo y las actitudes, se utilizó una Chi cuadrada de Pearson. Para el procesamiento de datos se utilizó el software SPSS v 21 .

\section{Resultados}

\section{Características sociodemográficas de la población estudiada}

De los 283 estudiantes encuestados, 215 (76\%) pertenecen al sexo femenino y 68 (24\%) son del sexo masculino; el promedio de edad en la población estudiada fue de $20.5 \pm 1.6$ años (rango de 18 a 24 años); $64.4 \%$ cursan de primero a cuarto semestre de la carrera; $94 \%$ de los estudiantes viven con su familia y de estos el $62.2 \%$ convive con un adulto mayor; $75.3 \%$ ha tratado temas sobre el adulto mayor, el 93.3\% le interesaría conocer más sobre el adulto mayor y el 51.9\% le gustaría desempeñarse en el área de la gerontología (Tabla 1).

Tabla 1. Características sociodemográficas de la población encuestada. $(n=283)$.

\begin{tabular}{|c|c|c|c|}
\hline Variable & Indicador & Frecuencia & Porcentaje \\
\hline \multirow{2}{*}{ Sexo } & Femenino & 215 & 76.0 \\
\hline & Masculino & 68 & 24.0 \\
\hline \multirow{3}{*}{ Edad } & $18-20$ & 153 & 54.0 \\
\hline & $21-23$ & 119 & 42.0 \\
\hline & $>24$ & 11 & 4.0 \\
\hline \multirow{4}{*}{ Grado escolar (Semestre) } & Primero - Segundo & 106 & 37.5 \\
\hline & Tercero - Cuarto & 76 & 26.9 \\
\hline & Quinto-Sexto & 54 & 19.1 \\
\hline & Séptimo - Octavo & 47 & 16.6 \\
\hline \multirow{4}{*}{ ¿Con quién vives? } & Familia & 266 & 94.0 \\
\hline & Solo & 11 & 3.9 \\
\hline & Amigos & 3 & 1.1 \\
\hline & Otro & 3 & 1.1 \\
\hline \multirow{3}{*}{ ¿En tu familia hay mayores de 60 años? } & $\mathrm{Si}$ & 176 & 62.2 \\
\hline & No & 104 & 36.7 \\
\hline & No sabe/no recuerda & 3 & 1.1 \\
\hline \multirow{2}{*}{ ¿Has vivido con mayores de 60 años? } & $\mathrm{Si}$ & 188 & 66.4 \\
\hline & No & 95 & 33.6 \\
\hline \multirow{2}{*}{ ¿En la carrera tratan temas sobre el AM? } & $\mathrm{Si}$ & 213 & 75.3 \\
\hline & No & 70 & 24.7 \\
\hline \multirow{2}{*}{ ¿Te interesaría conocer más acerca del AM? } & $\mathrm{Si}$ & 264 & 93.3 \\
\hline & No & 19 & 6.7 \\
\hline \multirow{2}{*}{ ¿Te gustaría desempeñarte en el área gerontológica? } & $\mathrm{Si}$ & 147 & 51.9 \\
\hline & No & 136 & 48.1 \\
\hline
\end{tabular}


Actitud de los estudiantes sobre los adultos mayores con

la escala de diferencial semántico de Osgood

La actitud hacia el AM, por parte del estudiante, mostró que ninguna de las variables positivas tuvo una puntuación superior al 50\%. Las de mayor puntaje reflejan que se percibe al adulto mayor como: valorado, protegido, sociable, e independiente; en cambio, las preguntas con menor puntaje indican que se percibe a esta población como poco hábil y poco resistente (Gráfica 1). En general la actitud que los estudiantes manifestaron tener hacia el adulto mayor es negativa con una media de 36.9 (DE \pm 5.8), con una diferencia de dos puntos porcentuales en relación con la positiva que tuvo una media de 37.7 (DE + 5.6). El 37\% tienen una actitud positiva, el 24\% una actitud neutral y el 39\% una actitud negativa. Cuando se determina la actitud del estudiante a partir de considerar las mejores actitudes de acuerdo a las 16 preguntas del cuestionario, se observa que no todos alcanzan los valores positivos, sin embargo, para este caso las mujeres tienen una mejor actitud hacia el AM que los hombres en una relación de 4:1; aunque no se observó ninguna relación de dependencia entre el sexo y las actitudes con Chi-cuadrada de Pearson (Gráfica 2).

Gráfica I. Percepción de los estudiantes de enfermería sobre las características de los adultos mayores con base en la Escala de diferencial semántico de Osgood.

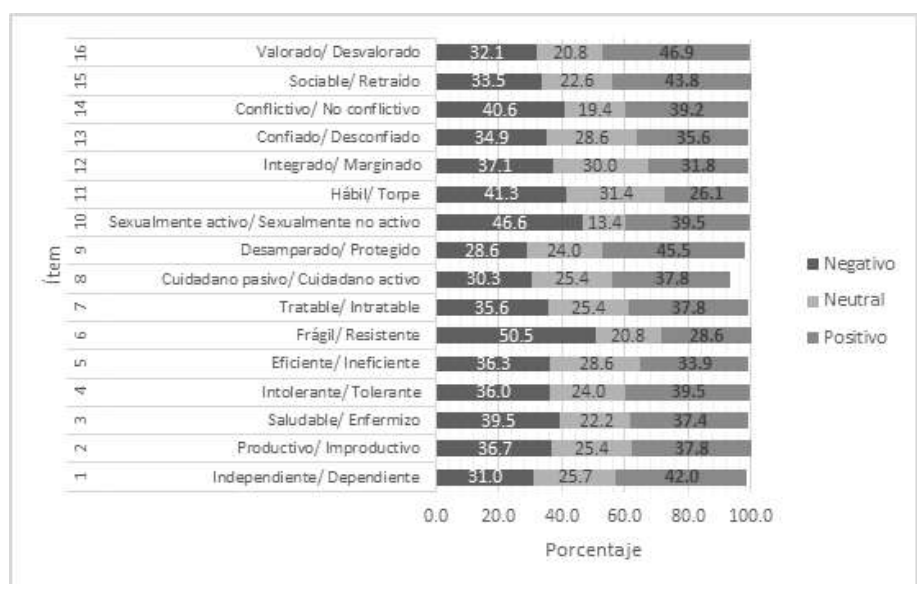

Gráfica 2. Distribución según género de la actitud de los estudiantes hacia el adulto mayor mediante la escala de diferencial semántico de Osgood.

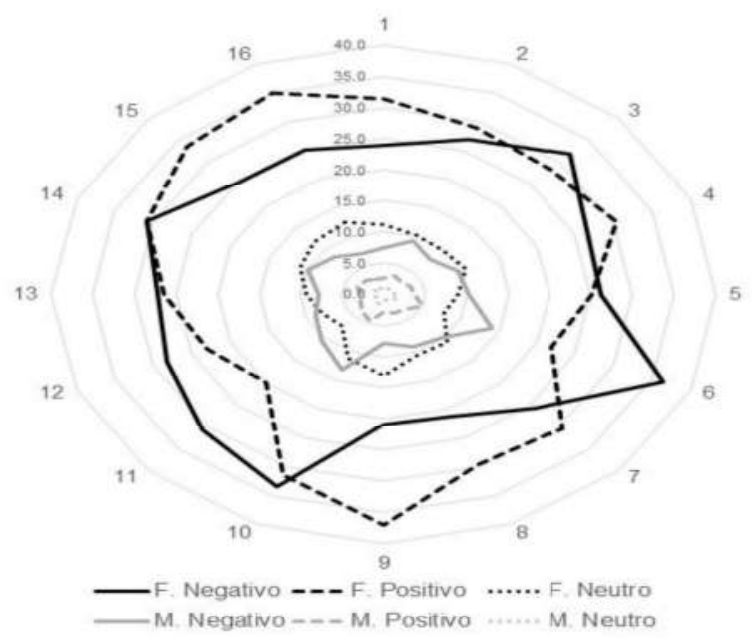


Actitud de los estudiantes sobre los adultos mayores con la escala Kogan

De los 204 puntos esperados, las respuestas se concentraron en el rango de puntaje que va de 105 a 139 puntos, seguido por el de 70 a 104, la media fue de 117.7 con una $\mathrm{DE} \pm 13.8$. La distribución de las respuestas se dividió en cuartiles, en el primero (0-25\%) se ubican aquellas iguales o menores a 109 puntos, en el segundo (26-50\%) de 110 a 118 puntos y en el tercero (51-75\%) de 119 a 128 puntos. Los puntajes se clasificaron en positivos y negativos a partir de la media, de acuerdo a esto, el $53 \%$ de los estudiantes tuvo una actitud positiva y el $47 \%$ una actitud negativa hacia el adulto mayor (Gráfica 3). En cuanto al género, una vez más se demostró que las actitudes positivas hacia el AM predominan en las mujeres con el 62\% y en los hombres sólo el $21.9 \%$.

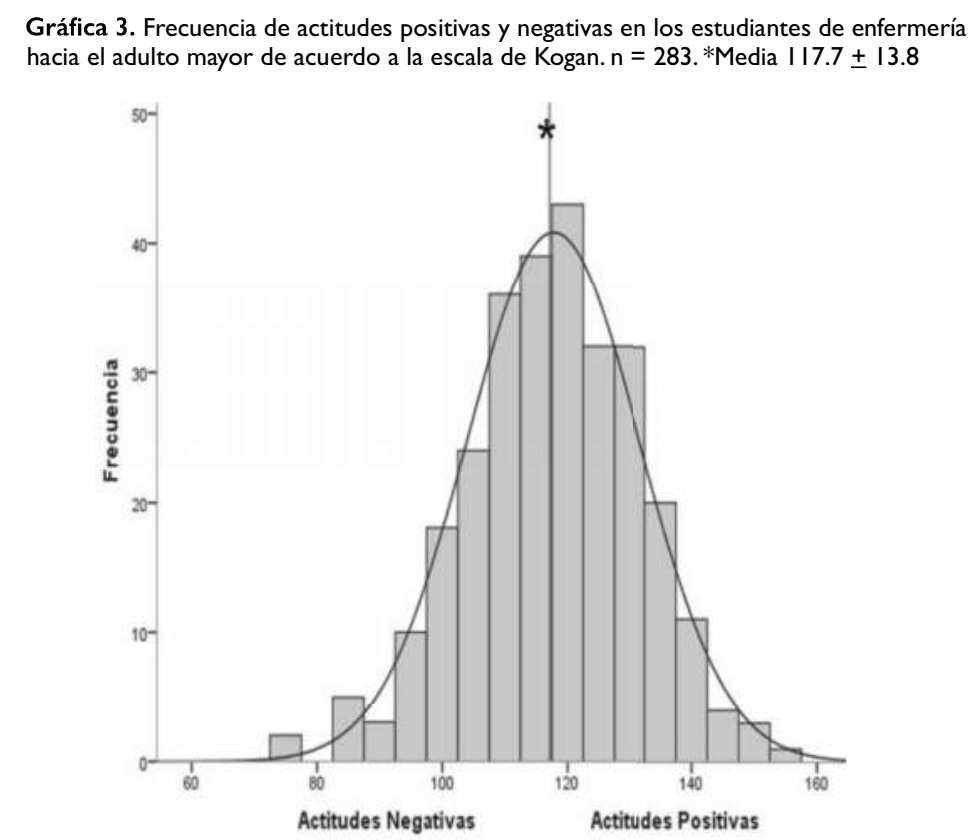

Al comparar los resultados sobre la actitud que tienen los estudiantes hacia los adultos mayores, entre la escala de diferencial semántico de Osgood y la escala de Kogan, se observan diferencias propias para cada tipo de estudio y situaciones ambivalentes. Tras utilizar la escala de Osgood existe mayor actitud negativa de los estudiantes relacionada, principalmente, a los estereotipos negativos del adulto mayor. Con la escala de Kogan la actitud del estudiante tiende a ser más positiva (Gráfica 4).

Gráfica 4. Comparación porcentual entre la escala de Osgood y Kogan sobre la actitud
que tienen los estudiantes de la licenciatura en enfermería hacia el adulto mayor.

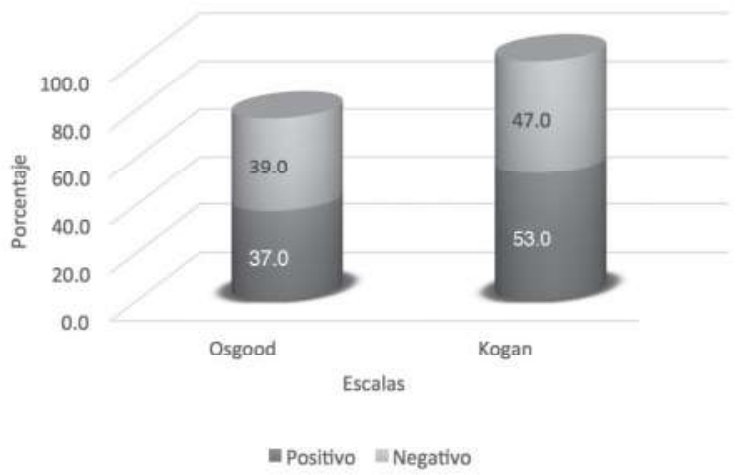




\section{Discusión}

Existen pocos estudios publicados sobre la actitud de los estudiantes de enfermería en México $^{23}$, y en ninguno se utilizó la escala de Kogan y Osgood. La opinión que tienen los estudiantes de la Licenciatura en Enfermería sobre los adultos mayores genera actitudes positivas y negativas. En el presente estudio la mayoría de los estudiantes cursaban los primeros semestres, con mayor prevalencia del sexo femenino.

A pesar de que un alto porcentaje de los estudiantes de enfermería convive con AM o han tratado temas sobre el adulto mayor, en la mayoría de ellos prevalecen las actitudes desfavorables y estereotipos negativos sobre los AM, posiblemente por la poca información o falta de conocimientos que tienen los estudiantes al inicio de su carrera; al avanzar en su carrera y tener mayor conocimiento, su interés y deseo por el cuidado del adulto mayor aumenta y un alto porcentaje, probablemente continuará con el cuidado de estos al término de su carrera, resultado similar a lo reportado en los Emiratos Árabes Unidos ${ }^{8}$; por otro lado, en estudiantes de enfermería españoles se reporta lo contrario ${ }^{9}$.

Por otra parte, las estudiantes tienen arraigado el instinto maternal de cuidadora, con mayor tendencia a los estereotipos positivos hacia los AM, estos resultados son consistentes con los reportados por Bernardini-Zambrini ${ }^{9}$ y Cheng-Chin et $\mathrm{a}^{10}$; sin embargo, existen estudios en países asiáticos en donde los hombres presentan mejor actitud que las mujeres hacia el $\mathrm{AM}^{8,20}$.

De acuerdo con Herdman ${ }^{24}$, la mayor prevalencia de actitudes negativas, pueden estar asociadas a factores culturales, religiosos, mitos y prejuicios que agravan el problema del envejecimiento en la población. En México existe una cultura y tradición familiar del cuidado del anciano al igual que en algunos países orientales ${ }^{8,10,20}$, además, la mujer juega un papel importante en el cuidado general de la familia, situación que posiblemente se relaciona a que en enfermería las mujeres tengan mejores actitudes hacia el paciente y los AM, por lo que es vital que los estudiantes adopten actitudes positivas desde el inicio de su carrera, sin importar el género.

La mayor parte de los estudiantes encuestados refirieron vivir en una familia en la que existen adultos mayores de 60 años, y son éstos estudiantes los que tienen mejor actitud hacia el AM, ninguna de las variables positivas tuvo una puntuación superior al 50\%. Las variables con mayor puntaje fueron: valorado, protegido, sociable, e independiente. Así también se puede observar que los ítems con menor puntaje resultaron hábil y resistente, resultados similares a los reportados por Haight et al ${ }^{25}$, donde los estudiantes que viven con sus abuelos tienen mejores actitudes hacia el AM, datos que contrastan con estudiantes de Holanda $\mathrm{y}^{7}$ de los Emiratos Árabes ${ }^{8}$, cuyos estudiantes otorgan mejores puntuaciones conforme avanzan en la carrera y adquieren mayores conocimientos sobre el adulto mayor.

Al medir la actitud de los estudiantes con la Escala de Diferencial Semántico de Osgood, ninguna de las variables positivas tuvo una puntuación superior a 50\%, se encontró una diferencia porcentual de dos puntos entre las actitudes negativas y positivas. Las variables con mayor puntaje fueron: valorado, protegido, sociable e independiente, y las preguntas con menor puntuación fueron hábil y resistente. Los datos son similares a los reportados por Duran-Badillo ${ }^{23}$ en estudiantes con un promedio de edad de 22 años, que cursaban los últimos semestres de la carrera de enfermería en Tamaulipas, México, en dicho estudio el 50.5\% de los estudiantes tenían estereotipos negativos ante la vejez.

En Chile, el Comité Nacional para el Adulto Mayor realizó durante el año 2002, un estudio sobre la imagen de la vejez de los estudiantes de enseñanza secundaria orientada a conocer la percepción sobre los adultos mayores ${ }^{26}$, el estudio determinó que existen mayores tendencias positivas hacia los adultos mayores por parte de las mujeres, sin embargo, la presencia de estereotipos negativos también fue evidente.

Las imágenes que los jóvenes construyen en torno a la vejez tienden a ser para la mayoría negativas, la juventud suele ser vista de manera positiva, mientras que la vejez se suele asociar con algo indeseable, atribuyéndoseles características negativas. No obstante, a nivel profesional, en el área de la salud, 
la actitud de los estudiantes de enfermería difiere de las actitudes de los estudiantes de medicina, ya que estos últimos tienen mejores actitudes hacia el $\mathrm{AM}^{27}$.

Al aplicar el instrumento de Kogan, los resultados obtenidos mostraron una relación inversa comparada con la de Osgood, ya que el mayor valor porcentual correspondió a las actitudes positivas, y en mayor medida en el sexo femenino, en este caso la diferencia encontrada fue de seis puntos porcentuales. Dato similar se observó en un estudio realizado en el personal de salud del primer nivel asistencial con una actitud positiva en el $53 \%$ de la población femenina ${ }^{15}$.

Con la escala de Osgood, la tendencia de los estudiantes fue hacia la actitud negativa con una diferencia de 2 puntos porcentuales sobre la positiva, lo que pudiera deberse a la complejidad de las preguntas en el cuestionario aplicado. De igual manera, los resultados coinciden con la investigación del Comité Nacional para el Adulto Mayor en Chile ${ }^{26}$. En lo referente a la pregunta si les gustaría desempeñarse en el área gerontológica, el 51.9\% le gustaría trabajar con el AM, comparado con el 75\% de los estudiantes en Salamanca, España ${ }^{9}$. Existen otros estudios con resultados similares a los aquí presentados, dirigida a estudiantes de medicina que tienden a desenvolverse mejor con el paciente geriátrico ${ }^{28}$.

La mayoría de los estudiantes encuestados posiblemente tengan una sobrevaloración de lo que hoy en día se tiene de su propia juventud, y de su comportamiento hacia las personas con las que convive. Los estereotipos y actitudes negativas están fuertemente marcados por evaluaciones negativas, debido a conceptos y atribuciones erróneas que tienen los jóvenes sobre la vejez, que pueden comprometer la calidad de la atención médica que reciban, mientras que una actitud positiva podrá mejorar la atención de salud hacia los adultos mayores. En una sociedad donde la realización personal se asocia al éxito, y éste es vinculado al logro de una mejor posición social y dinero, las capacidades competitivas, atribuidas mayoritariamente a los jóvenes y adultos jóvenes son vistas como objetivo central para acceder a la felicidad.

En México, los futuros profesionales en enfermería deben conocer el reglamento sobre la ley general de salud en materia de prestación de servicios de atención médica, mencionados en los artículos 29, 30 y 32, de los cuales se creó la carta de los derechos generales de las y los pacientes, que señala en sus artículos 3 y 9 el derecho a recibir información suficiente, clara, oportuna y veraz, así como el de contar con un expediente clínico apropiado, ya que el adulto mayor es sujeto prioritario de atención integral en materia de salud, tanto en los casos de emergencia médica como en enfermedades de carácter terminal ${ }^{29,30}$. Del mismo modo, es de suma importancia incluir o incrementar la cantidad y calidad en los programas académicos de la carrera de enfermería programas más amplios y completos con mayor interacción de los alumnos con el $\mathrm{AM}^{11,13}$.

\section{Limitaciones del Estudio}

Las limitaciones del presente estudio que deben tomarse en cuenta para la interpretación de los resultados son el pequeño tamaño de la muestra, la participación de estudiantes de una sola escuela de enfermería en el centro del país, por lo que los resultados no se pueden generalizar a otras escuelas de otros estados. Por otro lado, las escalas utilizadas han sido traducidas en varios idiomas y fueron elaboradas en la década de los sesenta y setenta, presentan ciertas limitaciones de acuerdo a las variables estudiadas por lo que podrían existir inconsistencias en su aplicación ${ }^{7,31-33}$ y comparación ${ }^{11}$. El presente estudio no comparó la actitud de los alumnos conforme avanzaban en su carrera, por lo tanto, es un compromiso para realizarse en futuros estudios. Sin embargo, podríamos considerar que las actitudes que tienen los estudiantes en toda la carrera son negativas, lo que invita a mejorar su conocimiento y actitudes hacia el adulto mayor.

\section{Conclusiones}

Las percepciones y actitudes hacia los AM ha sido objeto de estudio en múltiples investigaciones, desde el punto de vista de la psicología, la sociología y la educación. Al aplicar la escala de Osgood la actitud 
de los estudiantes tuvo una tendencia negativa. Con la escala de Kogan los resultados se tornaron positivos. Ambas escalas demostraron que las mujeres tienen una mejor actitud hacia el adulto mayor que los hombres.

Los resultados proveen información útil que podría ser considerada para futuros estudios, e identificar las razones que generan las actitudes de los estudiantes, ampliar la formación profesional del estudiante en enfermería con programas curriculares de capacitación y sensibilización sobre gerontología y geriatría, al aumentar su contacto profesional con los adultos mayores, sanos y enfermos, que contribuya a su labor sanitaria en cuanto al aprendizaje y trato con el AM, con el propósito de cambiar sus percepciones y actitudes de forma más positiva. Dicha capacitación tendría que ser brindada en los primeros semestres de pregrado y reconocer el valor de la geriatría en la educación de enfermería, que contribuya a mejorar la calidad de la atención holística que se otorga a un grupo poblacional que se incrementará dramáticamente en las siguientes décadas. Debido a que es un problema educativo, es necesario implementar estudios colaborativos entre las instituciones de enfermería, nacionales e internacionales, por lo tanto, buscar estrategias para preparar a los estudiantes en el futuro cercano.

\section{Responsabilidades éticas.}

Protección de personas y animales. Los autores declaran que para esta investigación no se han realizado experimentos en seres humanos ni en animales.

Confidencialidad de los datos. Los autores declaran que en este artículo no aparecen datos que permitan identificar a los participantes.

Derecho a la privacidad y consentimiento informado. Los autores han obtenido el consentimiento informado de los participantes.

Conflictos de interés: Los autores declaran no tener conflicto de intereses.

Declaración de Financiamiento: Financiado por el proyecto del Cuerpo Académico "Cuidado de la Salud en el Ciclo de Vida y Educación en Enfermería", PROMEP-UATLX-CA-210 de la Facultad de Ciencias de la Salud de la Universidad Autónoma de Tlaxcala, México.

Agradecimientos: Los autores agradecen a Erika Torres Tello por la revisión y traducción del manuscrito.

\section{Referencias}

1. Organización Mundial de la Salud. Envejecimiento y ciclo de vida. Datos interesantes acerca del envejecimiento. Ginebra: OMS; 2015.

2. Gutiérrez Robledo LM, Téllez-Rojo MM, Manrique Espinoza B, et al. Encuesta Nacional de Salud y Nutrición 2012 Evidencia para la política pública en salud. Discapacidad y dependencia en adultos mayores mexicanos: un curso sano para una vejez plena. México: Instituto Nacional de Salud Pública, Secretaria de Salud; 2012.

3. Consejo Nacional de Población. Dinámica Demográfica 1999-2010 y Proyecciones de Población 2010-2030 Tlaxcala. México: CONAPO; 2014.

4. Ruiz Guerrero JA. La transición demográfica y el envejecimiento poblacional: futuros retos para la política de salud en México. Encrucijada. Revista electrónica del Centro de Estudios en Administración Pública. 2011;2(8):1-16.

5. Aguayo C, Nass L. Estereotipos sociales como amenaza a la inserción de adultos mayores. Revisión narrativa. J Oral Res. 2013; 2(3): 145-52. http://dx.doi.org/10.17126/JORALRES.2013.032

6. Ruelas-González MG, Pelcastre-Villafuerte BE, Reyes-Morales H. Maltrato institucional hacia el adulto mayor: percepciones del prestador de servicios de salud y de los ancianos. Salud pública Mex. 2014; 56(6): 631-7. 
7. Bleijemberg N, Jansen MJM, Schuurmans MJ. Dutch nursing students' knowledge and attitudes towards older people - A longitudinal cohort study. J Nurs Educ Pract. 2012; 2(2): 1-8.

https://doi.org/10.5430/jnep.v2n2p1

8. Sheikh RB, Mathew E, Rafique AM, et al. Attitude of medical students toward old people in Ajman, United Arab Emirates. Asian J Gerontol Geriatr. 2013; 8(2): 85-9.

9. Bernardini Zambrini DA, Moraru M, Hanna M, et al. Attitudes toward the elderly among students of health care related studies at the University of Salamanca, Spain. J Contin Educ Health Prof. 2008; 28(2): 86-90. http://dx.doi.org/10.1002/chp.162

10. Wang CC, Liao WC, Kao MC, et al. Taiwanese medical and nursing student interest levels in and attitudes towards geriatrics. Ann Acad Med Singapore. 2009; 38(3): 230-6.

11. Engström G, Fagerberg I. Attitudes towards older people among Swedish health care students and health care professionals working in elder care. Nurs Rep. 2011; 1(1): 1-6.

https://doi.org/10.4081/nursrep.2011.e2

12. Fishbein M, Ajzen I. Belief, attitude, intention, and behavior: An introduction to theory and research. San Francisco: Addison-Wesley Pub. Co; 1975.

13. Deschodt M, de Casterlé BD, Milisen K. Gerontological care in nursing education programmes. J Adv Nurs. 2010; 66(1): 139-48. http://dx.doi.org/10.1111/j.1365-2648.2009.05160.x

14. Ortíz PJ, Tello T, Pérez ME, et al. Actitudes hacia la atención del adulto mayor por parte del personal que labora en los servicios de emergencia en hospitales de Lima. Acta Med Per. 2013; 30(2): 74-8.

15. Sampén JD, Varela LF, Díaz C, et al.Validación de la Escala de Actitudes hacia el Adulto Mayor de Kogan y evaluación de las Actitudes hacia el adulto mayor por parte del personal de salud del primer nivel asistencial. Acta Med Per. 2012; 29(3): 147-54.

16. Llanes C. La transculturación en el cuidado de enfermería gerontológica comunitaria. Rev Cubana Enfermer. 2012; 28(3): 195-208.

17. Kogan N. Attitudes towards old people: The development of a scale and examination of correlates. J Abnormal Soc Psychol. 1961; 62(1): 44-54.

18. Osgood CE, Suci GJ, Tannenbaum PH. El diferencial semántico cómo instrumento de medida. En: Wainerman C, Stevens S, Thorndike RL, et al. Escalas de medición en ciencias sociales. Buenos Aires: Nueva Visión; 1976. p. 331-69.

19. Despaigne M, Jiménez E, Martínez B. Intervención educativa sobre violencia a integrantes de la Universidad del Adulto Mayor “24 de Febrero”. MEDISAN. 2011; 15(1): 105-11.

20. Liu Z, Pu L, Wang H, et al. Survey of attitude towards and understanding of the elderly amongst Chinese undergraduate medical students. Asian Biomed. 2014; 8(5): 615-22.

https://doi.org/10.5372/1905-7415.0805.335

21. World Medical Association. World Medical Association Declaration of Helsinki: ethical principles for medical research involving human subjects. JAMA. 2013; 310(20): 2191-4. http://dx.doi.org/10.1001/jama.2013.281053

22. Secretaría de Salud. Norma Oficial Mexicana NOM-012-SSA3-2012, Que establece los criterios para la ejecución de proyectos de investigación para la salud en seres humanos. México: SSA; 2012.

23. Duran-Badillo T, Miranda-Posadas C, Cruz-Barrera LG, et al. Estereotipos negativos sobre la vejez en estudiantes universitarios de enfermería. Rev Enferm Inst Mex Seguro Soc. 2016; 24(3): 205-9.

24. Herdman E. Challenging the discourses of nursing ageism. Int J Nurs Stud. 2002; 39(1):105-14.

25. Haight BK, Christ MA, Djas JK. Does nursing education promote ageism? J Adv Nurs. 1994; 20(2): 382-90.

26. Arnold-Cathalifaud M, Thumala D, Urquiza A, et al. La vejez desde la mirada de los jóvenes chilenos: estudio exploratorio. Ultima décad. 2007; (27): 75-91. 
27. Bernardini DA, Moraru M, Macías-Núñez JF. ¿Son modificables las actitudes hacia las personas mayores en estudiantes de medicina? Experiencia en la Universidad de Salamanca. Educ méd. 2009; 12(2): 111-5.

28. Martina M, Gutiérrez C, Mejia M, et al. Percepción del estudiante de medicina de una universidad pública acerca del docente adulto mayor y del adulto mayor en general. An. Fac. med., Univ. nac. mayor San Marcos. 2014; 75(3): 237-44.

29. Secretaría de Salud. Norma Oficial Mexicana NOM-168-SSA1-1998, del expediente clínico. MéxiCO: SSA; 1998.

30. Secretaria de Salud. Carta de los derechos generales de los pacientes. México: SSA; 2001.

31. Harris DK, Changas PS, Palmore EB. Palmore's first facts on aging quiz in a multiple-choice format. Educ Gerontol. 1996; 22(6): 575-89. http://dx.doi.org/10.1080/0360127960220605

32. Pennington HR, Pachana NA, Coyle SL. Use of the facts on aging quiz in New Zeland: Validation of questions, perfomance of a student sample, and effects of a don't know option. Educ Gerontol. 2001; 27(5): 409-16. https://doi.org/10.1080/03601270152053438

33. Polizzi KG, Millikin RJ. Attitudes toward the elderly: Identifying problematic usage of ageist and overextended terminology in research instructions. Educ Gerontol. 2002; 28(5): 367-77. http://dx.doi.org/10.1080/03601270290081344 